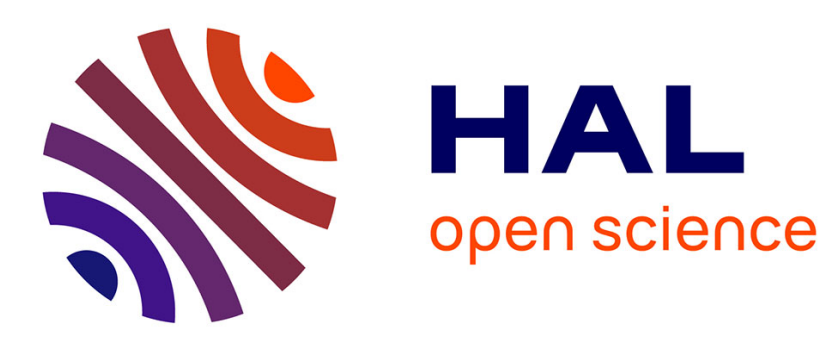

\title{
iQAS: An Integration Platform for QoI Assessment as a Service for Smart Cities
}

\author{
Antoine Auger, Ernesto Expósito, Emmanuel Lochin
}

\section{To cite this version:}

Antoine Auger, Ernesto Expósito, Emmanuel Lochin. iQAS: An Integration Platform for QoI Assessment as a Service for Smart Cities. 3rd IEEE World Forum on Internet of Things, WF-IoT 2016, Reston, VA, USA, December 12-14, 2016, Dec 2016, Reston, United States. pp.88-93, 10.1109/WFIoT.2016.7845400 . hal-01908068

\section{HAL Id: hal-01908068 https://hal-univ-pau.archives-ouvertes.fr/hal-01908068}

Submitted on 24 Jan 2020

HAL is a multi-disciplinary open access archive for the deposit and dissemination of scientific research documents, whether they are published or not. The documents may come from teaching and research institutions in France or abroad, or from public or private research centers.
L'archive ouverte pluridisciplinaire HAL, est destinée au dépôt et à la diffusion de documents scientifiques de niveau recherche, publiés ou non, émanant des établissements d'enseignement et de recherche français ou étrangers, des laboratoires publics ou privés. 


\title{
iQAS: An Integration Platform for QoI Assessment as a Service for Smart Cities
}

\author{
Antoine Auger ${ }^{\dagger}$, Ernesto Exposito ${ }^{\ddagger}$ and Emmanuel Lochin ${ }^{\dagger}$ \\ ${ }^{\dagger}$ Institut Supérieur de l'Aéronautique et de l'Espace (ISAE-SUPAERO), \\ Université de Toulouse, 31055 Toulouse Cedex 4, France \\ \{antoine.auger, emmanuel.lochin\}@isae.fr \\ ${ }_{\ddagger}$ Laboratoire Informatique de l’Université de Pau et des Pays de l’Adour (LIUPPA) \\ ernesto.exposito@univ-pau.fr
}

\begin{abstract}
While reducing costs and improving sustainability, a common goal for Smart Cities is to become more "liveable" for their citizens. By taking advantage of new information sources offered by the Internet of Things (IoT), cities can rely on sensing platforms to improve their service offer. These sensing platforms, however, raise new research challenges, in particular regarding Quality of Information (QoI). To cope with this issue, common platforms generally provide quality-oriented internal mechanisms. Nevertheless, the configuration of such platforms is complex, especially for Smart City stakeholders that may have various skill levels and different areas of expertise. As a result, QoI assessment is often delegated to end applications where developers have to implement their own adaptation mechanisms.

This paper proposes and describes iQAS, an integration platform for QoI Assessment as a Service for Smart Cities. iQAS is autonomic, extensible and configurable, allowing Smart City stakeholders to collaboratively assess and improve (when possible) QoI in real-time. While the platform development is at its early stages, we illustrate within a concrete case study the need for QoI assessment and the benefits to implement adaptation mechanisms.
\end{abstract}

Keywords-Quality of Information; Autonomic Computing; integration platform; Smart City; Cloud; Internet of Things

\section{INTRODUCTION}

In an annual report issued in 2014, United Nations (UN) predicts that the urban population will increase of 2.4 billion over next 40 years. The same report projects that, in 2050, 66\% of the world's population will be urban [1]. To support such a massive growth, cities will be forced to adapt themselves and evolve in a sustainable way. We believe that Smart Cities and their sensing platforms can be a key aspect of this transformation.

These sensing platforms raise new research challenges, such as the one of the Quality of Information (QoI). Broadly speaking, QoI measures the fitness-of-use of information (by relying on attributes such as accuracy, cost and timeliness) for a given application in a specific context. However, the understanding and the implementation of the QoI notion generally require domain-specific skills (e.g., weather forecast). As a result, Smart City stakeholders may poorly understand the importance of considering QoI within sensing platforms. Despite this, end information consumers (mainly citizens or applications) may require QoI-specific needs. In this case, even if they do not have the required skills, developers have to design and implement their own adaptation mechanisms. This may oblige developers to implement the same mechanism many times, within each of their applications. To cope with this QoI challenge, this paper proposes and describes an integration platform for QoI Assessment as a Service (iQAS). Autonomic, extensible and configurable, iQAS is a tool to assess and improve (when possible) QoI in a collaborative way.

The rest of this paper is structured as follows: Section II introduces the required background, dealing with QoI, Sensing as a Service and Autonomic Computing notions. Then, Section III motivates the need and lists the requirements for the iQAS platform, which is then described in section Section IV. Finally, Section V presents related work while Section VI concludes and describes further implementation perspectives.

\section{REQUIRED BACKGROUND}

In this section the main basis of our proposal will be presented. In particular, the iQAS platform requires a minimal background in QoI, integration platforms and self-management properties guaranteed by the Autonomic Computing paradigm.

\section{A. Quality of Information}

For Smart City stakeholders, the design of informationcentric platforms comes with new research challenges closely linked to information quality [2]. Indeed, these platforms provide services by assuming that information received from sources is reliable and of better quality than that required by end consumers. Unfortunately, this is not always the case and sensing platforms may provide information that does not really satisfy consumer needs. Considered for a while, common Quality of Service (QoS) metrics have shown limitations to characterise and evaluate information quality [3]. In practice, QoS mostly refers to network packet transportation. As a result, network QoS is no longer suitable to characterize information required by a given information consumer within a specific context.

Quality of Information (QoI) has been introduced to extend the commonly-used QoS metrics (bandwidth, delay, jitter and losses), which were too restrictive. In [3], Bisdikian et al. defined QoI as "the body of tangible evidence available (i.e., the innate information properties) that can be used to make 
judgments about the fitness-of-use and utility of information products". QoI has influenced the definition of recent ontologies for IoT. Some of them, such as the one of Wang et al. [4] or the Quality Ontology [5], provide QoI support through the definition of accuracy, cost and timeliness concepts.

\section{B. Sensing as a Service}

Cloud Computing has promoted the Everything as a Service (XaaS) model [6]. In addition to Infrastructure as a Service (IaaS), Platform as a Service (PaaS) and Software as a Service (SaaS) [7], we have recently witnessed the birth of Sensing as a Service (SeaS) [8]. This sensing model consists in taking advantage of certain features of cloud-based platforms (pay as you go, elasticity, multi-tenancy, Service Level Agreements, etc.) while considering distinct entities and stakeholders involved in the sensing process.

Smart City platforms play a major role in the integration of heterogeneous information sources, various services and different stakeholders. Therefore, we advocate that these sensing platforms fall within the service model of "integration Platform as a Service" (iPaaS). Indeed, according to Gartner, an iPaaS is a "suite of cloud services enabling development, execution and governance of integration flows connecting any combination of on premises and cloud-based processes, services, applications and data within individual or across multiple organizations" [9].

\section{Autonomic Computing}

Autonomic Computing has been defined by IBM as the ability for systems to "manage themselves given high-level objectives from administrators" [10]. Since this paradigm makes a clear distinction between goals and means, the Autonomic Computing paradigm is commonly considered as a convenient way to build interoperable, lasting and easy-to-use systems.

By definition, autonomic systems are a set of Autonomic Elements. Each of these elements is composed of one or many Managed Elements controlled by a single Autonomic Manager. The latter continuously monitors the internal state of its different Managed Elements; then analyses this information; and finally takes appropriate decisions based on both its knowledge base and high-level objectives. At last, these decisions are converted into actions and transmitted to appropriate Managed Elements for execution. These different steps form the MAPE-K adaptation control loop (Monitor, Analyse, Plan, Execute, Knowledge base), also denoted as "MAPE-K loop" in the rest of this paper.

\section{MOtivATION AND PLATFORM REQUIREMENTS}

\section{A. Problem statement}

A Smart City generally provides sensing functionality to its different stakeholders [11]. In this paper, we consider four different categories of stakeholders, namely Information and Communication Technologies (ICT) experts, domain experts, public managers and citizens [12]. Regarding iQAS usage, we envision citizens as information consumers only, without considering neither crowdsourcing nor crowdsensing use case.
These stakeholders may have various interests that lead to various QoI requirements. Besides, they may have different skill levels and different areas of expertise. This can be an issue within common sensing platforms, excluding some people who are unable to take part in the implementation of the QoI feature. Indeed, these people may even be unable to express basic QoI requirements, due to a lack of knowledge about this notion. This challenge motivates the present contribution, which aims at proposing a QoI-oriented tool to help Smart City actors to understand, improve and assess QoI according to their skills and interests.

Main requirements for the design of the iQAS platform will be given in the next section. Please note that iQAS mainly focuses on QoI assessment and QoI-related adaptation mechanisms. In particular, the process of data acquisition (using protocols such as CoAP for instance) is out of the scope of this proposal.

\section{B. $i Q A S$ requirements}

Prior any development or implementation phase, we defined key requirements for the iQAS platform. To not reinvent the wheel, we reuse some requirements that have already been identified in the literature. In particular, we found particularly helpful:

1) technical reports and specifications produced by the Focus Group on Smart Sustainable Cities (FG-SCC) of ITU-T $^{1}$

2) deliverables of the EU FP7 CityPulse project ${ }^{2}$;

3) deliverables of the Internet of Things Architecture (IoT-A) project ${ }^{3}$.

We make the distinction between functional $(\mathrm{F})$ and nonfunctional (NF) requirements. In total, we considered 8 requirements, listed below:

F1 Stakeholders should be able to select or define QoI attributes of interest;

F2 Stakeholders should be able to perform sensing queries and assess QoI in real-time;

F3 Stakeholders should be able to enable or disable specific adaptation mechanisms on the fly;

F4 Stakeholders should be able to define their own information workflows;

NF1 The platform should be usable and configurable by stakeholders with different skills;

NF2 The platform should be extensible by supporting addition of new services or new information sources;

NF3 The platform should be easy to maintain;

NF4 The platform should be scalable.

\section{INTEGRATION PLATFORM PROPOSAL}

\section{A. iQAS architecture}

In this section, we present the high-level architecture of the iQAS platform. As depicted in Figure 1, the iQAS architecture

\footnotetext{
${ }^{1}$ http://www.itu.int/en/ITU-T/focusgroups/ssc

${ }^{2}$ http://www.ict-citypulse.eu/page/content/publications

${ }^{3} \mathrm{http}: / /$ www.iot-a.eu/public/public-documents
} 
follows microservices approach [13]. This choice has been motivated by the necessity to have a non-monolithic cloudbased architecture. Furthermore, such an architecture will permit extensibility and scalability. In the following, we detail some key features of the iQAS platform and we highlight how they address the requirements previously drawn.

1) Information ingress: the role of adapters is to allow the binding of various information sources. At first, the platform provides a set of quick-start adapters to ICT experts. However, these latter still have the possibility to develop and integrate new ones according to their needs. As a result, the use of this design pattern allows the binding of various information sources (including raw datasets). This addresses the extensibility requirement NF2.

The Information collection service is in charge of the mapping between input stream data and internal information representation within the iQAS platform. It also holds capabilities and characteristics of the different information sources. As a possible next step, we plan to allow stakeholders to define several granularities of information (such as Raw Data, Information, Knowledge and Wisdom [14]). Besides, since many datasets are already semantically annotated, we also consider the possibility to add semantic support.

The QoI annotation service is a key component within the iQAS platform. It allows the computation and the annotation of each observation with QoI attributes. At first, only some QoI attributes will be proposed to stakeholders (such as provenance, timeliness or trust). Then, we plan to allow the computation of new QoI attributes, based on the content of information (e.g., trust may vary according to provenance) or some information fields (e.g., timeliness may be computed using the timestamp value). This addresses the functional requirement $\mathbf{F 1}$.

2) Adaptation and monitoring: the Adaptation service consists in a repository containing the different mechanisms that can be applied to either information stream or to a piece of information (e.g., filtering mechanism, prediction mechanism, etc.). These mechanisms are similar to mathematical functions. They may have several inputs and outputs. For instance, a domain expert user (e.g., a meteorologist) can design and add a filtering mechanism without having a deep knowledge or understanding of the iQAS platform. Then, this mechanism is available for others stakeholders who can define new information workflows and MAPE-K adaptation rules.

The iQAS platform has a teaching objective by allowing stakeholders to monitor the impact of their settings (adaptation mechanisms, rules, information workflows, etc.) on QoI (F2). As a result, we seek this platform to help them to better understand QoI notion and its inherent challenges. This specific objective has motivated the need for being able to import an already existing dataset into iQAS in order to run multiple experiments with different configurations.

Finally, the Monitoring service provides detailed statistics through a web user interface. In real-time, stakeholders can see the QoI evolution when changing sensor configuration, the number of information sources or enabling/disabling some adaptation mechanisms (F3). The Monitoring service also provides detailed statistics regarding each process of the MAPE-K loop (such as symptoms and plans for instance).

3) Configuration, extensibility and scalability: sensing platforms generally rely on complex monolithic architectures with many dependencies between their components. Within iQAS, there was an attempt to separate the three main functionalities of the platform, namely information processing, adaptation and monitoring features. As a result, each stakeholder can now focus on the configuration or the monitoring of some components according to its area of expertise. This addresses requirements NF1 and $\mathbf{F 4}$.

When correctly documented, a microservices architecture is easily maintainable (NF3) and extensible (NF2). Let us imagine the case where an administrative consultant needs to receive e-mail notifications in case of heat wave. With the microservices approach, such service can be easily developed into the iQAS platform by taking advantage of existing functionalities (monitoring or sensing services for instance). In order to complete the service integration, ICT experts just have to make appropriate bindings with MAPE-K loop and expose the final Notification service through the API gateway.

Scalability of the iQAS platform will be considered in a second time. However, we are confident that a cloudbased microservices architecture is suitable to achieve at least vertical scalability (using cloud elasticity). If needed, horizontal scalability (using load balancers for instance) will be considered to meet the requirement NF4.

\section{B. iQAS stakeholders and use cases}

As stated earlier, we consider four different stakeholder categories: ICT experts, domain experts, public managers and citizens. For each kind of stakeholders, Table I gives an overview of their areas of expertise and examples of use cases. While ICT experts have skills in computer science, networking or data processing; domain experts have domain-specific knowledge (e.g., meteorologists) or expertise in policies and other processes (e.g., security expert). Public managers are composed of administrative staff of the city (such as the mayor and consultants) and other company managers (e.g., public transportation).

Figure 2 shows typical steps for the use of the iQAS platform. First, domain experts should help ICT experts with the binding of information sources and the configuration of the QoI annotation process (step $\mathbf{0}$ in Figure 2). In particular, skills of domain experts will be appreciable to configure the QoI annotation process, selecting relevant QoI attributes or defining new ones. Then, Smart City stakeholders can configure the iQAS platform. For instance, after domain experts have defined adaptation mechanisms, ICT experts can use them in their information workflows. Public managers can also define high-level business rules to receive alerts, reports and other statistical information (step (2). Once started and running, the 


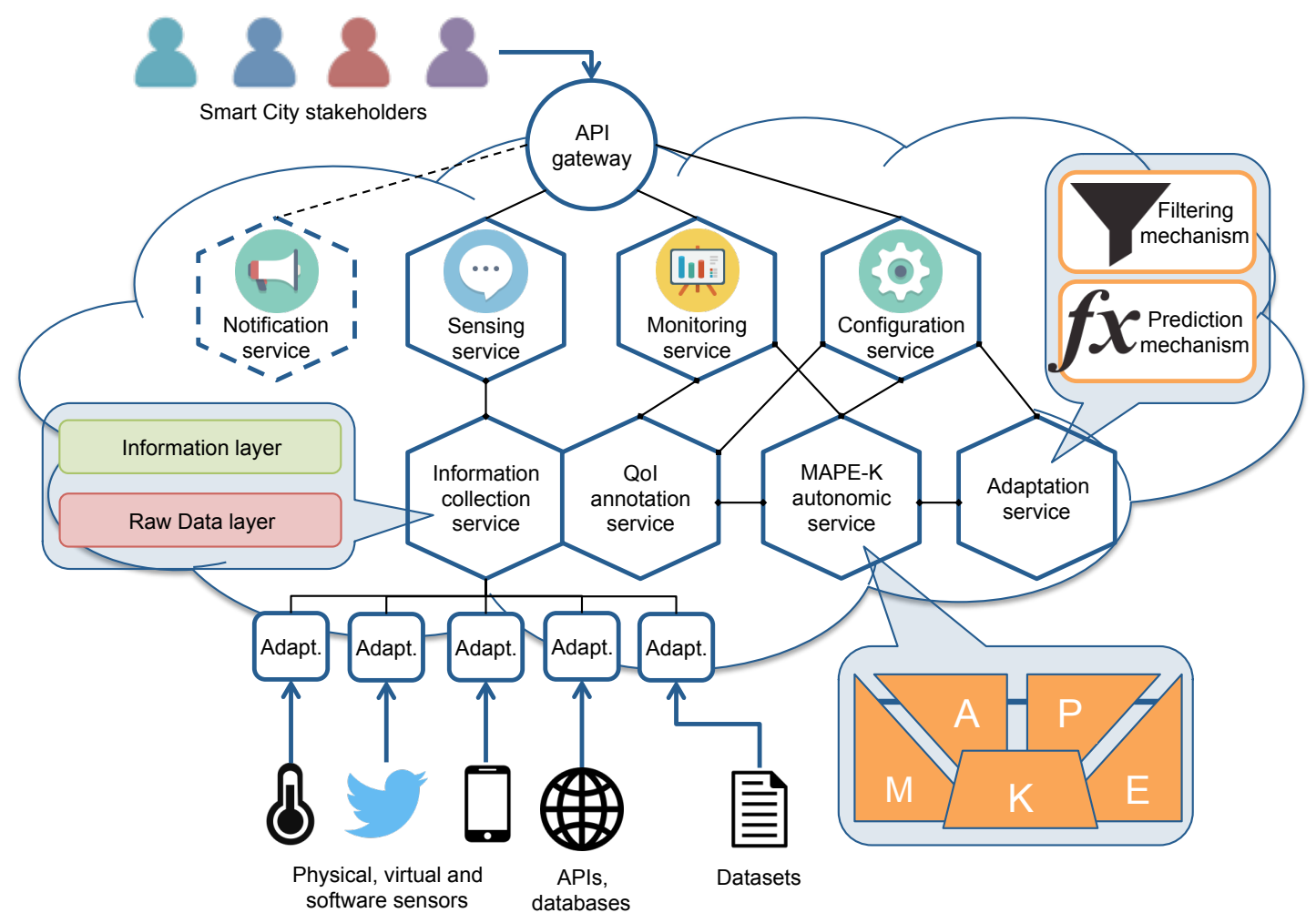

Fig. 1: Integration platform for QoI Assessment as a Service (iQAS)

\begin{tabular}{cll}
\hline $\begin{array}{c}\text { Smart City } \\
\text { stakeholders }\end{array}$ & Areas of expertise & \multicolumn{1}{c}{ Use cases } \\
\hline ICT experts & $\begin{array}{l}\text { Computer science, } \\
\text { networking, infor- } \\
\text { mation processing }\end{array}$ & $\begin{array}{l}\text { Configuration: binding of in- } \\
\text { formation sources, definition of } \\
\text { information workflows } \\
\text { Monitoring: QoI assessment, } \\
\text { MAPE-K monitoring }\end{array}$ \\
Domain experts & $\begin{array}{l}\text { Domain-specific } \\
\text { knowledge, } \\
\text { policies, processes }\end{array}$ & $\begin{array}{l}\text { Configuration: sensor configu- } \\
\text { ration, QoI and semantic anno- } \\
\text { tation, definition of adaptation } \\
\text { mechanisms } \\
\text { Monitoring: QoI assessment }\end{array}$ \\
Public managers & $\begin{array}{l}\text { City management, } \\
\text { legal responsibility }\end{array}$ & $\begin{array}{l}\text { Configuration: definition of } \\
\text { business rules } \\
\text { Monitoring: alerts, reports and } \\
\text { statistics }\end{array}$ \\
& & $\begin{array}{l}\text { Sensing as a Service (environ- } \\
\text { ment, public transportation, etc.) }\end{array}$ \\
Citizens & Not applicable &
\end{tabular}

TABLE I: Examples of use cases for the iQAS platform according to stakeholder skills

iQAS platform can process incoming queries coming from the development team (private testing) or citizens (public testing) (step (3). Finally, the iQAS platform provides real-time monitoring feature to ICT experts, domain experts and public managers. According to their roles and skills, the stakeholders have access to custom dashboards through a web-based user interface (step (4).

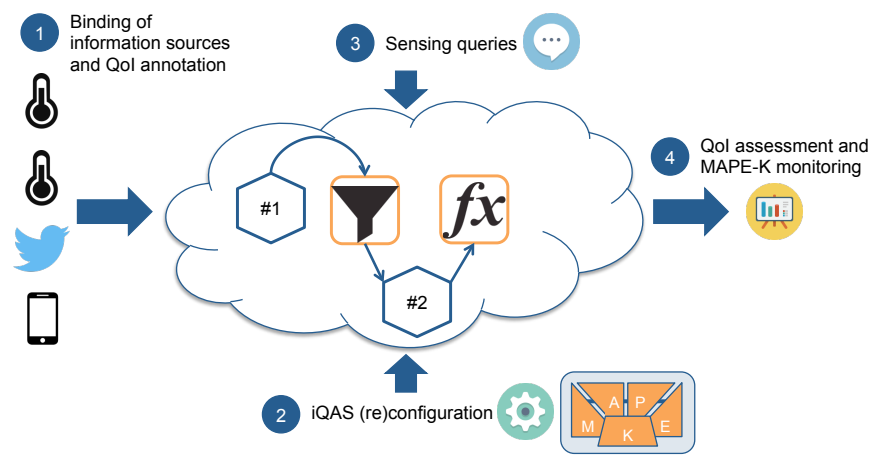

Fig. 2: Typical steps for the use of the iQAS platform

\section{Concrete case study}

We developed a first prototype of the iQAS platform to demonstrate the need for QoI assessment and the benefits to implement adaptation mechanisms. For convenience, we downloaded a raw weather dataset recorded in the city of Aarhus in Denmark ${ }^{4}$. In particular, we only processed the visibility measurements collected from February 2014 to June 2014.

We bound this information source to the iQAS platform thanks to a specific CityPulse adapter. Then, we configured the iQAS platform itself. First, we declared this adapter to the Information collection service. We configured the QoI

\footnotetext{
${ }^{4}$ http://iot.ee.surrey.ac.uk:8080/datasets.html\#weather
} 
annotation service to annotate all observations received from the CityPulse adapter with a "provenance" QoI attribute. In our case, we considered that all observations came from a single physical sensor that we denoted as sensor 1 . Regarding adaptation mechanisms, we developed a basic filtering mechanism and declared it available to the Adaptation service. We also declared the "visibility" concept into the Knowledge base of the MAPE-K autonomic service, as well as the information sources that might report this kind of observations (the sensor 1 in this case). For the "visibility" concept, we set the observation range to $[0,+\infty]$ (in meteorology, the visibility is always a positive distance measurement).

At this point, the configuration of the platform was finished and the adaptation process could take place. The MAPE-K autonomic service monitored all information that came in the platform and inferred its type (temperature, humidity, visibility, etc.) thanks to the "provenance" attribute and its Knowledge base. Then, this service systematically applied the filtering mechanism to check that the received observations matched the properties and capabilities of the corresponding information sources. When a value did not satisfy them (e.g., a negative value coming from sensor 1 which reports "visibility" measurements), it was simply discarded.

Using different configurations, we processed the same dataset twice with the help of the iQAS platform. The first time, we disabled all adaptation mechanisms (see Figure 3a). The second time, we enabled adaptation mechanisms and filtering mechanism (see Figure 3b). Thanks to the Monitoring service, we discovered that the observation range of the raw visibility dataset was in the interval $[-10000,50]$, with 331 records reporting a value of -9999.0 kilometers. Many reasons could be envisioned to explain these "fake measurements". However, the fact that these records only concerned a single precise value ( -9999.0 kilometers) has led us to conclude to systematic errors during the sensing process [15]. To be noted that both analysis and adaptation processes would have been much more complicated to perform if QoI management was delegated to end applications.

This simple example clearly illustrates the rationale of this paper to develop a QoI-oriented integration platform for Smart Cities. More than ever, sensing platforms require highlevel QoI to provide acceptable services to citizens. When possible, adaptation mechanisms may be used to improve information quality and therefore quality of the offered service. In particular, the use of such mechanisms is particularly relevant to deal with communication-based issues (end-to-end delay, connectivity losses, etc.) and sensor-based errors.

\section{RELATED WORK}

QoI has recently gained attention, in particular within IoT domain. For instance, OpenIoT [16] is an open source cloud solution for the IoT that rely on a publish/subscribe middleware. Even if this solution provides pre-filtering of sensor data based on high-level requirements, it is mainly focused on application development and do not allow the user to add its own adaptation mechanisms. In a more abstract way, the IoT-A project [17] has delivered an architectural reference model for IoT. This framework proposes to use a secure network encryption protocol to tackle QoI issue. However, this approach only takes into account transport-based errors and malicious attacks, without considering the intrinsic information quality nor sensor-based errors for instance.

Up to now, far too little attention has been paid to the application of the Autonomic Computing paradigm to Smart Cities. In [18], Pujolle proposed an autonomic architecture based on QoS adaptation for VoIP between things. However, this solution does not deal with QoI and cannot be applied to sensing platforms. Other cloud-based commercial solutions rely on MAPE-K loop (e.g., IBM Watson IoT [19]) or allow the user to define information workflows and rules (e.g., AWS IoT [20]). Nevertheless, these proprietary solutions are application-oriented and do not provide any QoI insights. Besides, the extensibility and the customization of these platforms are quite limited.

Combining works within QoI and IoT research fields, researchers have tried to address specific Smart City challenges. SmartSantander [21] is an experimental platform based on a large number of IoT devices deployed within the Santander city. The main goal of this project is to provide reusable services for application development (outdoor parking management, environment monitoring, participatory sensing, etc.). ALMANAC [22] is a federated Smart City platform that aims to integrate several information sources (IoT devices, capillary and metro access networks) and several existing Smart City platforms to offer richer services to citizens. This project focuses on semantic interoperability, end-to-end security and privacy. Regarding adaptation mechanisms, it provides data fusion feature by using complex event processing. Just as the DIMMER platform [23], our solution has been designed following a microservices architecture. Finally, closer to our iQAS proposal, the CityPulse framework [24] aims to provide large-scale stream processing solutions for Smart City applications. This framework considers QoI (with the computation of collection point-related Key Performance Indicators) and QoI inspection (with tools such as the CityPulse QoI Explorer). Differently from CityPulse, iQAS relies on an autonomic MAPE-K adaptation control loop and allows easy implementation of new adaptation mechanisms. Compared to most of the monolithic sensing platforms for Smart Cities, the iQAS platform is extensible, configurable and usable by stakeholders with different skills and interests.

\section{CONCLUSIONS AND PERSPECTIVES}

In this paper, we have presented the requirements and the architecture of an integration platform for QoI Assessment as a Service (iQAS) within a Smart City context. While the platform development is at its early stages, a concrete case study has shown the need for QoI assessment and the benefits to implement adaptation mechanisms. This evaluation has been performed based on a raw visibility dataset, recorded in the city of Aarhus, Denmark. To the best of our knowledge, the iQAS platform is the first attempt bringing together 


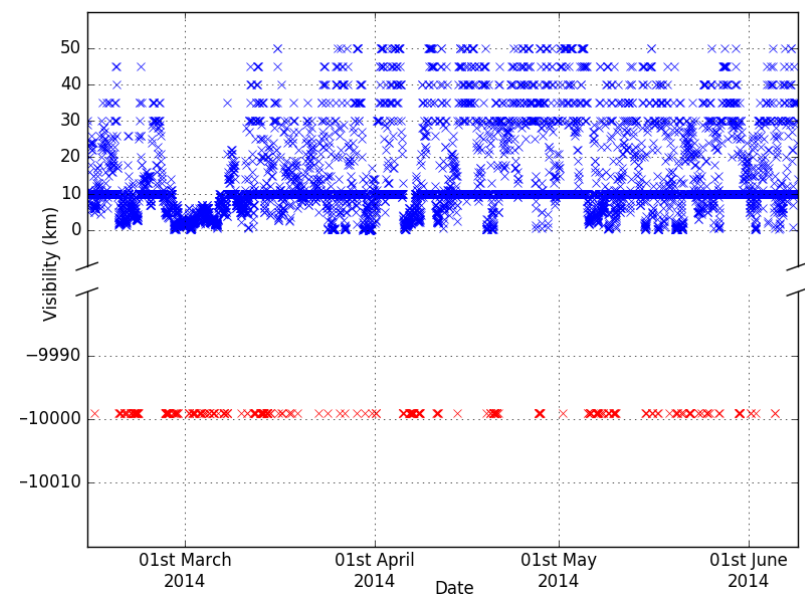

(a) Without any adaptation mechanisms

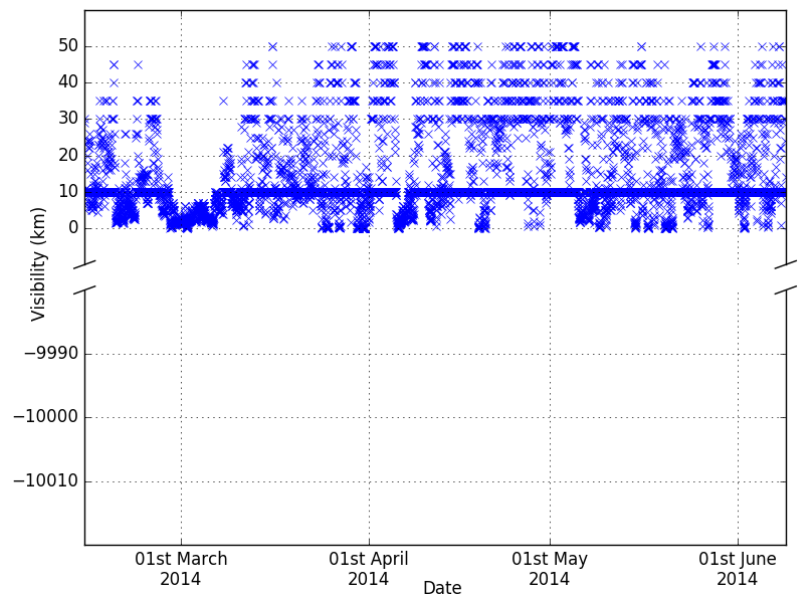

(b) With filtering mechanism enabled: observation range set to $[0,+\infty]$ for the "visibility" concept

Fig. 3: Measured visibility (km) in Aarhus, Denmark from February 2014 to June 2014

QoI, Sensing as a Service and the Autonomic Computing paradigms. With this proposal, our motivation is to help the different Smart City stakeholders to assess, better understand and improve QoI in a collaborative way.

As future work, we plan to refine the implementation of the platform before performing more in-depth QoI experiments. In particular, we will study the relevance of certain technologies such as Apache nifi or the OASIS framework for Topology and Orchestration Specification for Cloud Applications (TOSCA), with respect to iQAS requirements.

\section{ACKNOWLEDGEMENT}

This research was supported in part by the French Ministry of Defense through financial support of the Direction Générale de l'Armement (DGA).

\section{REFERENCES}

[1] United Nations, World Urbanization Prospects 2014: Highlights. United Nations Publications, 2014.

[2] P. Barnaghi, M. Bermudez-Edo, and R. Tönjes, "Challenges for Quality of Data in Smart Cities," J. Data and Information Quality, vol. 6, no. 2-3, pp. 6:1-6:4, 2015.

[3] C. Bisdikian, L. M. Kaplan, and M. B. Srivastava, "On the Quality and Value of Information in Sensor Networks," ACM Trans. Sen. Netw., vol. 9, no. 4, pp. 48:1-48:26, 2013.

[4] W. Wang, S. De, R. Toenjes, E. Reetz, and K. Moessner, "A Comprehensive Ontology for Knowledge Representation in the Internet of Things," in 2012 IEEE 11th International Conference on Trust, Security and Privacy in Computing and Communications (TrustCom), Jun. 2012, pp. 1793-1798.

[5] Marten Fischer, Thorben Iggena, and Daniel Kümper, "Quality Ontology Working Draft," Feb. 2016. [Online]. Available: https: //mobcom.ecs.hs-osnabrueck.de/cp_quality/

[6] P. Banerjee, R. Friedrich, C. Bash, P. Goldsack, B. Huberman, J. Manley, C. Patel, P. Ranganathan, and A. Veitch, "Everything as a service: Powering the new information economy," Computer, no. 3, pp. 36-43, 2011.

[7] S. Patidar, D. Rane, and P. Jain, "A survey paper on cloud computing," in Advanced Computing \& Communication Technologies (ACCT), 2012 Second International Conference on. IEEE, 2012, pp. 394-398.
[8] C. Perera, A. Zaslavsky, P. Christen, and D. Georgakopoulos, "Sensing as a Service Model for Smart Cities supported by Internet of Things," Transactions on Emerging Telecommunications Technologies, vol. 25, no. 1, pp. 81-93, 2014.

[9] M. Pezzini and B. J. Lheureux, "Integration Platform as a Service: moving integration to the Cloud," Gartner, 2011.

[10] J. O. Kephart and D. M. Chess, "The vision of autonomic computing," Computer, vol. 36, no. 1, pp. 41-50, 2003.

[11] G. P. Hancke, G. P. Hancke Jr et al., "The role of advanced sensing in smart cities," Sensors, vol. 13, no. 1, pp. 393-425, 2012.

[12] G. Lepouras, C. Vassilakis, C. Halatsis, and P. Georgiadis, "Domain expert user development: The SmartGov approach," Communications of the ACM, vol. 50, no. 9, pp. 79-83, 2007.

[13] S. Newman, Building Microservices. O'Reilly Media, Inc., 2015.

[14] A. Sheth, "Internet of Things to Smart IoT Through Semantic, Cognitive, and Perceptual Computing," IEEE Intelligent Systems, vol. 31, no. 2, pp. 108-112, 2016.

[15] J. R. Taylor and E. R. Cohen, "An introduction to error analysis: the study of uncertainties in physical measurements," Measurement Science and Technology, vol. 9, no. 6, p. 1015, 1998.

[16] EU FP7 project, "OpenIoT: Open Source cloud solution for the Internet of Things." [Online]. Available: http://www.openiot.eu/

[17] — "IOT-A: Internet of Things Architecture." [Online]. Available: http://www.iot-a.eu/public

[18] G. Pujolle, "An Autonomic-oriented Architecture for the Internet of Things," in IEEE John Vincent Atanasoff 2006 International Symposium on Modern Computing (JVA'06), Oct. 2006, pp. 163-168.

[19] IBM, "Watson IoT." [Online]. Available: http://www.ibm.com/ internet-of-things/

[20] Amazon Web Services, "AWS IoT." [Online]. Available: https: //aws.amazon.com/fr/iot/

[21] L. Sanchez, L. Muñoz, J. A. Galache, P. Sotres, J. R. Santana, V. Gutierrez, R. Ramdhany, A. Gluhak, S. Krco, E. Theodoridis et al., "SmartSantander: IoT experimentation over a smart city testbed," Computer Networks, vol. 61, pp. 217-238, 2014.

[22] D. Bonino, M. T. D. Alizo, A. Alapetite, T. Gilbert, M. Axling, H. Udsen, J. A. C. Soto, and M. Spirito, "ALMANAC: Internet of Things for Smart Cities," in 2015 3rd International Conference on Future Internet of Things and Cloud (FiCloud), 2015, pp. 309-316.

[23] A. Krylovskiy, M. Jahn, and E. Patti, "Designing a Smart City Internet of Things Platform with Microservice Architecture," in 2015 3rd International Conference on Future Internet of Things and Cloud (FiCloud), 2015, pp. 25-30.

[24] D. Puiu, P. Barnaghi, R. Tönjes, D. Kümper, M. I. Ali, A. Mileo, J. X. Parreira, M. Fischer, S. Kolozali, N. Farajidavar et al., "CityPulse: Large Scale Data Analytics Framework for Smart Cities," IEEE Access, vol. 4, pp. 1086-1108, 2016. 